\title{
Infinite generation of the kernels of the Magnus and Burau representations
}

\author{
THOMAS CHURCH \\ BENSON FARB
}

\begin{abstract}
Consider the kernel $\mathrm{Mag}_{g}$ of the Magnus representation of the Torelli group and the kernel Bur $_{n}$ of the Burau representation of the braid group. We prove that for $g \geq 2$ and for $n \geq 6$ the groups $\mathrm{Mag}_{g}$ and $\mathrm{Bur}_{n}$ have infinite rank first homology. As a consequence we conclude that neither group has any finite generating set. The method of proof in each case consists of producing a kind of "Johnson-type" homomorphism to an infinite rank abelian group, and proving the image has infinite rank. For the case of $\operatorname{Bur}_{n}$, we do this with the assistance of a computer calculation.
\end{abstract}

20F34, 20F36, 57M07

\section{Introduction}

\subsection{The Magnus kernel}

Let $S:=S_{g, 1}$ be a compact, connected, oriented surface of genus $g \geq 2$ with one boundary component. Let $\operatorname{Mod}_{g, 1}$ denote the mapping class group of $S$, which is the group of homotopy classes of orientation-preserving homeomorphisms of $S$ which fix $\partial S$ pointwise. Let $\mathcal{I}_{g, 1}$ denote the Torelli group, which is the subgroup of $\operatorname{Mod}_{g, 1}$ consisting of elements that act trivially on $H:=H_{1}(S, \mathbb{Z})$.

The group $\operatorname{Mod}_{g, 1}$ acts on the fundamental group $\pi_{1}(S)$, inducing an action on the solvable quotient $\Gamma / \Gamma^{3}$, where $\Gamma:=\pi_{1}(S), \Gamma^{2}=[\Gamma, \Gamma]$ and $\Gamma^{3}=\left[\Gamma^{2}, \Gamma^{2}\right]$ are the first three terms of the derived series of $\Gamma$. In this paper we consider the group

$$
\operatorname{Mag}_{g}:=\operatorname{ker}\left(\operatorname{Mod}(S) \rightarrow \operatorname{Aut}\left(\Gamma / \Gamma^{3}\right)\right) .
$$

It follows from work of Fox [4, Theorem 4.9] that $\mathrm{Mag}_{g}$ coincides with the kernel of the so-called Magnus representation (see Birman [2, Chapter 3])

$$
r: \mathcal{I}_{g, 1} \rightarrow \mathrm{GL}_{2 g}(\mathbb{Z} H) .
$$

The group $\operatorname{Mag}_{g}$ is called the Magnus kernel. It was an open question for some time whether or not $\operatorname{Mag}_{g}$ is nontrivial. This was settled in the affirmative by Suzuki in [12]. The first main result of this paper is that $\mathrm{Mag}_{g}$ is in fact quite large. 
Theorem 1.1 For $g \geq 2$ the group $H_{1}\left(\operatorname{Mag}_{g}, \mathbb{Z}\right)$ has infinite rank.

As the abelianization of a finitely-generated group has finite rank, we deduce the following.

Corollary 1.2 For $g \geq 2$ the group $\operatorname{Mag}_{g}$ has no finite generating set.

The idea of our proof of Theorem 1.1 is to define a kind of "Johnson-type" homomorphism (see Johnson [5]):

$$
\Psi: \operatorname{Mag}_{g} \rightarrow \operatorname{Hom}\left(G^{\mathrm{ab}}, \bigwedge^{2} G^{\mathrm{ab}}\right)
$$

where $G=[\Gamma, \Gamma]$ and $G^{\mathrm{ab}}$ denotes the abelianization of $G$. We then construct infinitely many linearly independent elements contained in the image.

It will follow from the definition of $\Psi$ that $\Psi$ extends to $\operatorname{Mag}\left(F_{n}\right)$, the "Magnus kernel" for $\operatorname{Aut}\left(F_{n}\right)$. Thus as an immediate corollary we obtain that $\operatorname{Mag}\left(F_{n}\right)$ is not finitely generated. Since the first posting of this paper, a different proof of this last result has been given by Satoh [11]. Satoh's approach shows that the image of $\operatorname{Mag}\left(F_{n}\right)$ under $\Psi$ has abelian quotients of arbitrarily large finite rank.

\subsection{The Burau kernel}

Let $B_{n}$ denote the braid group on $n$ strands. $B_{n}$ can be realized (see Section 4 below) as a subgroup of the automorphism group $\operatorname{Aut}\left(F_{n}\right)$ of the free group of rank $n$. The Burau representation is a homomorphism

$$
\rho_{n}: B_{n} \rightarrow \mathrm{GL}_{n}\left(\mathbb{Z}\left[t, t^{-1}\right]\right) .
$$

We define the Burau kernel, denoted $\operatorname{Bur}_{n}$, to be the kernel of $\rho_{n}$. Let $K$ be the kernel of the homomorphism $F_{n} \rightarrow \mathbb{Z}$ taking each fixed generator of $F_{n}$ to 1 . It follows easily from Fox [4] that

$$
\operatorname{Bur}_{n}=\operatorname{ker}\left(B_{n} \rightarrow \operatorname{Aut}\left(F_{n} /[K, K]\right)\right) .
$$

While $\rho_{3}$ is faithful, it was a longstanding problem as to whether or not $\rho_{n}$ is faithful (that is, whether $\operatorname{Bur}_{n}$ is nontrivial) for $n>3$. This was solved by Moody [9], LongPaton [7], and Bigelow [1] in various cases, with the result that $\operatorname{Bur}_{n}$ is nontrivial for $n \geq 5$; the case of $n=4$ is still open. Our next main result is that $\operatorname{Bur}_{n}$ is in fact quite large for $n \geq 6$.

Theorem 1.3 For $n \geq 6$ the group $H_{1}\left(\operatorname{Bur}_{n}, \mathbb{Z}\right)$ has infinite rank; in particular, $\operatorname{Bur}_{n}$ has no finite generating set. 
To prove Theorem 1.3 we construct, similarly to the proof of Theorem 1.1 above, a homomorphism

$$
\Phi: \operatorname{Bur}_{n} \rightarrow \operatorname{Hom}\left(K^{\mathrm{ab}}, \bigwedge^{2} K^{\mathrm{ab}}\right) .
$$

The elements which have been constructed in the kernel of the Burau representation are geometrically elegant, but algebraically very complicated; for example, the element of Bur $_{7}$ found by Long-Paton can be described by a single diagram, but as a free group automorphism sends generators of $F_{7}$ to words of length up to 475137. Thus we need the assistance of a computer in order to calculate $\Phi$ explicitly (see Section 4 below for a full discussion). For the computations in this paper we use a simpler element $\phi_{B} \in \operatorname{Bur}_{n}$ for $n \geq 6$ found by Bigelow, which takes generators to words of length no more than 9841 . Once we compute the form of $\Phi\left(\phi_{B}\right)$, we then use an equivariance property of $\Phi$ to prove that the image of $\Phi$ has infinite rank, from which Theorem 1.3 follows.

We remark that in [10, Problem 6.24] Morita posed the problem of determining the kernel of the Magnus and Burau (among other) representations. Theorem 1.1 and Theorem 1.3 can be viewed as a partial answer to this problem.

Acknowledgements We are grateful to William Goldman, whose Mathematica notebook FreeGroupAutos.nb was very helpful in our computations of the expression in Appendix A. We would like to thank Dan Margalit and Tam Nguyen Phan for careful comments on an earlier version of this paper. We also thank Mark Kidwell for a historical correction. The second author gratefully acknowledges support from the National Science Foundation.

\section{Defining the homomorphisms}

The following construction works for any group $G$ whenever one considers automorphisms of the universal 2-step nilpotent quotient $G / G_{3}$ acting trivially on the abelianization $G^{\mathrm{ab}}$. Johnson [5] considered the case $G=\Gamma=\pi_{1}(S)$.

With $\Gamma$ equal to $\pi_{1}(S)$ or $F_{n}$ as in the introduction, we take $G:=[\Gamma, \Gamma]$ or $G:=K$ respectively. In either case, let $G_{i}$ be the lower central series of $G$, defined inductively by $G_{1}=G$ and $G_{i+1}=\left[G, G_{i}\right]$. Consider the exact sequence

$$
1 \rightarrow G_{2} \rightarrow G \rightarrow G^{\mathrm{ab}} \rightarrow 1 .
$$

Centralizing (1) gives

$$
1 \rightarrow G_{2} / G_{3} \rightarrow G / G_{3} \rightarrow G^{\mathrm{ab}} \rightarrow 1
$$


Since $G$ is free, taking (1) as a presentation for $G^{\mathrm{ab}}$, Hopf's formula gives that

$$
G_{2} / G_{3} \approx \bigwedge^{2} G^{\mathrm{ab}}
$$

$\operatorname{Aut}(\Gamma)$ acts on $\Gamma$, and thus on $G$, and the isomorphism $v: G_{2} / G_{3} \approx \bigwedge^{2} G^{\text {ab }}$ respects the action of $\operatorname{Aut}(\Gamma)$ on both sides. In particular, conjugation by $\Gamma$ descends to an action on $G^{\text {ab }}$ by $H=\Gamma /[\Gamma, \Gamma]$ or by $\mathbb{Z}=\Gamma / K$ respectively. In the case $G=[\Gamma, \Gamma]$, the fact that $\operatorname{Mag}_{g}$ acts trivially on $\Gamma / \Gamma^{3}$ implies that $\operatorname{Mag}_{g}$ acts trivially on $G^{\text {ab }}=\Gamma^{2} / \Gamma^{3}$ and on $\bigwedge^{2} G^{\mathrm{ab}}$. Similarly, in the case $G=K$, we have that $\operatorname{Bur}_{n}$ acts trivially on $G^{\mathrm{ab}}$ and on $\bigwedge^{2} G^{\mathrm{ab}}$.

Let $f \in \operatorname{Mag}_{g}$ (respectively, $f \in \operatorname{Bur}_{n}$ ) be given. For $x \in G^{\text {ab }}$, pick any lift $\tilde{x} \in G$. Since $f$ acts trivially on both the quotient and kernel of (2), we see that $f(\tilde{x}) \tilde{x}^{-1}$ lies in the kernel $G_{2} / G_{3}$, which we identify with $\bigwedge^{2} G^{\text {ab }}$ via the isomorphism above. One checks, exactly as in Johnson [5], that

$$
\delta_{f}: G^{\mathrm{ab}} \rightarrow \bigwedge^{2} G^{\mathrm{ab}}
$$

defined by $\delta_{f}(x):=f(\tilde{x}) \tilde{x}^{-1}$ is a well-defined homomorphism; in fact, the resulting map $\delta_{f}$ is $\mathbb{Z} H$-linear (respectively, $\mathbb{Z}\left[t, t^{-1}\right]$-linear) with respect to the conjugation action on $G^{\mathrm{ab}}$. This is equivalent to the claim that

$$
\delta_{f}\left(\gamma x \gamma^{-1}\right) \equiv \gamma \delta_{f}(x) \gamma^{-1} \bmod G_{3},
$$

which can be checked as follows. The difference between the left and right side is

$$
\left(f\left(\gamma x \gamma^{-1}\right) \gamma x^{-1} \gamma^{-1}\right)\left(\gamma f(x) x^{-1} \gamma^{-1}\right)^{-1}=f(\gamma) f(x) f(\gamma)^{-1} \gamma f(x)^{-1} \gamma^{-1},
$$

which is conjugate to $\left[\gamma^{-1} f(\gamma), f(x)\right]$. The condition on $f$ implies that $f(\gamma) \equiv$ $\gamma \bmod G_{2}$, so $\gamma^{-1} f(\gamma) \in G_{2}$ and $\left[\gamma^{-1} f(\gamma), f(x)\right] \in G_{3}$ as desired.

One also checks, exactly as in [5], that in the case $G=[\Gamma, \Gamma]$, defining the map $\Psi$ by $\Psi(f):=\delta_{f}$ gives a well-defined homomorphism;

$$
\Psi: \operatorname{Mag}_{g} \rightarrow \operatorname{Hom}\left(G^{\mathrm{ab}}, \bigwedge^{2} G^{\mathrm{ab}}\right) .
$$

and, in the case $G=K$, defining $\Phi(f):=\delta_{f}$ gives a well-defined homomorphism:

$$
\Phi: \operatorname{Bur}_{n} \rightarrow \operatorname{Hom}\left(G^{\mathrm{ab}}, \bigwedge^{2} G^{\mathrm{ab}}\right) .
$$

The homomorphisms $\Psi$ and $\Phi$ are equivariant with respect to the natural actions of $\operatorname{Aut}(\Gamma)$ on the source and target. 


\section{Computing the image of $\Psi$}

Let $S_{0,4}$ denote the 2 -sphere with 4 open disks removed. A lantern in $S$ is an embedding $S_{0,4} \hookrightarrow S$. Consider the two simple closed curves $\alpha$ and $\beta$ and the three $\operatorname{arcs} A_{1}, A_{2}$ and $A_{3}$ on $S_{0,4}$ given in Figure 1 .
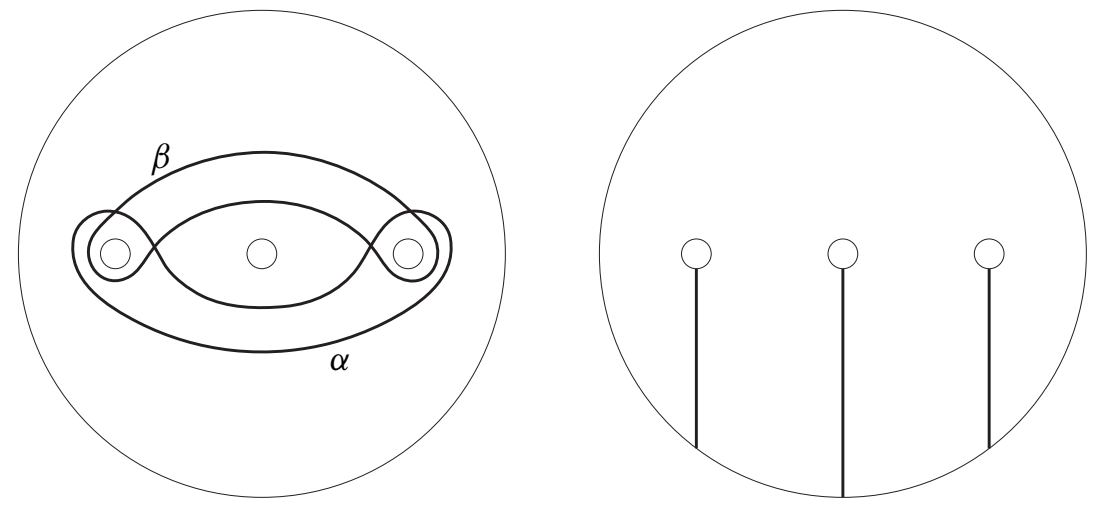

Figure 1: The simple closed curves $\alpha$ and $\beta$, and the $\operatorname{arcs} A_{1}, A_{2}, A_{3}$

One directly computes the action of $f:=T_{\alpha} T_{\beta}^{-1}$ on $A_{1}, A_{2}$ and $A_{3}$, as follows (see Figure 2). Let $x, y$, and $z$ be the loops which begin with $A_{1}, A_{2}$ and $A_{3}$, respectively, go clockwise around the appropriate boundary component of $S_{0,4}$, then come back along the same arc $A_{i}$. Let $X, Y, Z$ be the inverses of $x, y, z$ in $\pi_{1}\left(S_{0,4}\right)$. Then:

$$
\begin{aligned}
& f\left(A_{1}\right)=x y X z x Y X Z A_{1}=[x y X, z] A_{1} \\
& f\left(A_{2}\right)=Z X z x A_{2}=[Z, X] A_{2} \\
& f\left(A_{3}\right)=Z X z x Y X Z x z x y X A_{3}=[Z X z, x Y X] A_{3}
\end{aligned}
$$

Let $L$ be an embedding of a lantern in $S$ with the property that each of the four boundary curves of $L$ are separating in $S .{ }^{1}$ In this case we can observe that $T_{\alpha} T_{\beta}^{-1} \in \operatorname{Mag}_{g}$, as follows. Note that the elements corresponding to $x, y, z$ all lie in $\Gamma^{2}$. Furthermore, $\Gamma=\pi_{1}(S)$ has a basis where each element $c$ is either disjoint from $L$, or else of the form $c=A \gamma A^{-1}$, where $A$ is an arc intersecting $L$ in some $A_{i}$ and $\gamma$ is a loop disjoint from $L$. In the former case the element $f=T_{\alpha} T_{\beta}^{-1}$ fixes $c$. In the latter case, assume for example that $A$ intersects $L$ in $A_{2}$; then we have

$$
f(c)=f\left(A \gamma A^{-1}\right)=f(A) \gamma f(A)^{-1}=[Z, X] A \gamma A^{-1}[X, Z]=[Z, X] c[X, Z]
$$

${ }^{1}$ To formally identify $x, y, z$ with elements of $\Gamma=\pi_{1}(S)$, we choose a basepoint on $\partial S$, and arcs from this basepoint to $L$ meeting $L$ in one point. Since $f$ is the identity off of $L$, any ambiguity in the choice of these paths to $L$ does not affect the computation. 
Since $x, y, z \in \Gamma^{2}$, we have $[Z, X] \in \Gamma^{3}$; thus $f(c) \equiv c \bmod \Gamma^{3}$. The same is true for $A_{1}$ and $A_{3}$, so we conclude that $f(c) \equiv c \bmod \Gamma^{3}$ for all elements of a basis for $\Gamma$, implying $T_{\alpha} T_{\beta}^{-1} \in \operatorname{Mag}_{g}$. Suzuki gave a more illuminating proof that elements of this form lie in $\operatorname{Mag}_{g}$ in [13].
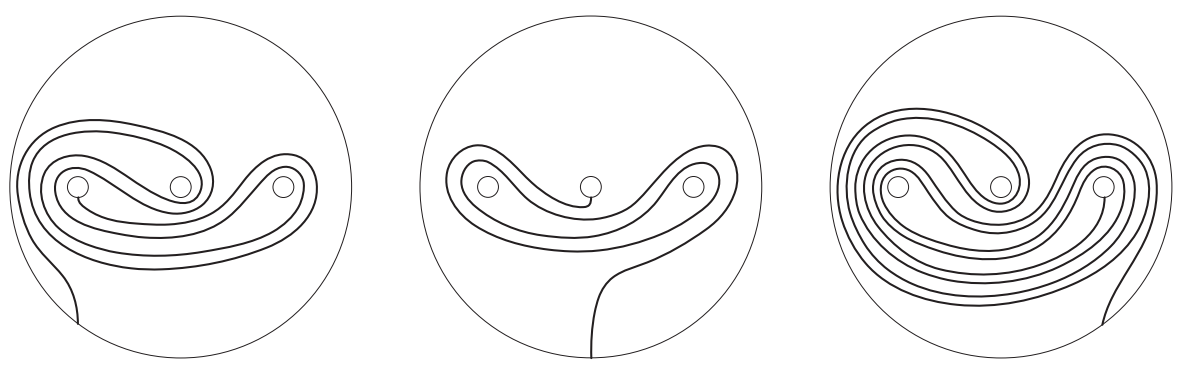

Figure 2: The arcs $f\left(A_{1}\right), f\left(A_{2}\right)$ and $f\left(A_{3}\right)$

We are now ready to compute $\Psi$. For $a, b \in \Gamma$, we denote by $\{a, b\}$ the image in $G^{\text {ab }}$ of $[a, b] \in G$ under the abelianization map.

Proposition 3.1 Let $L$ be a lantern embedded in $S$ so that each of the four boundary curves of $L$ are separating in $S$. Let $a$ and $b$ be loops intersecting $L$ in $A_{1}$ and $A_{2}$. Then

$$
\Psi\left(T_{\alpha} T_{\beta}^{-1}\right)(\{a, b\})=(a-1)(b-1)[x \wedge z+y \wedge z] .
$$

Note that the right hand side of (5) is an element of $\bigwedge^{2} G^{\text {ab }}$, considered as a $\mathbb{Z} H-$ module, and the action of $a$ and $b$ on this module factors through $H$.

Proof As in the computation above, we have

$$
f([a, b])=[f(a), f(b)]=[w a, v b]
$$

where

$$
w=[[x y X, z], a] \text { and } v=[[Z, X], b] .
$$

From the assumption on the embedding of $L$ we have $x, y, z \in G$, and thus $w, v \in G_{2}$. We will use the following commutator identities, which hold in any group; we write ${ }^{x} y$ for $x y x^{-1}$.

$$
[w a, b]={ }^{w}[a, b][w, b] \quad[a, v b]=[a, v]^{v}[a, b]
$$

We then find that

$$
[w a, v b]={ }^{w}[a, v]{ }^{w v}[a, b][w, v]^{v}[w, b]
$$


Note that the second term lies in $G$, the first and fourth in $G_{2}$, and the third in $G_{3}$.

We want to compute $f([a, b])[a, b]^{-1}$ as an element of the quotient $G_{2} / G_{3}$. Note that $[w, v] \equiv 0 \bmod G_{3}$, and that conjugating an element of $G$ by an element of $G_{2}$ is a trivial operation modulo $G_{3}$. Finally, since $[[a, b],[w, b]] \in G_{3}$, we can move $[a, b]$ to the right to cancel $[a, b]^{-1}$. We thus obtain

$$
\begin{aligned}
f([a, b])[a, b]^{-1} & ={ }^{w}[a, v]{ }^{w v}[a, b][w, v]^{v}[w, b][a, b]^{-1} \\
& \equiv[a, v][a, b][w, b][a, b]^{-1} \bmod G_{3} \\
& \equiv[a, v][w, b] \bmod G_{3} .
\end{aligned}
$$

Recall that the action of $\Gamma$ on $\Gamma$ by conjugation descends to a $\mathbb{Z} H$ action on $G^{\text {ab }}$. Recall from above the isomorphism $v: G_{2} / G_{3} \rightarrow \bigwedge^{2} G^{\mathrm{ab}}$. Since the homology class of $x$ is trivial in $H$, we have

$$
v([x y X, z])=y \wedge z \text { and } v([Z, X])=z \wedge x .
$$

It follows that

$$
v(w)=v([[x y X, z], a])=(1-a) y \wedge z
$$

and

$$
v(v)=v([[Z, X], b])=(1-b) z \wedge x .
$$

We therefore have that

$$
v([a, v][w, b])=(a-1) v-(b-1) w=(a-1)(1-b) z \wedge x-(b-1)(1-a) y \wedge z .
$$

We conclude that

$$
\Psi\left(T_{\alpha} T_{\beta}^{-1}\right)(\{a, b\})=(a-1)(b-1)[x \wedge z+y \wedge z]
$$

as desired.

Theorem 3.2 The image of $\Psi$ has infinite rank for $g \geq 3$.

Proof Let $\gamma$ and $\delta_{k}$ be the curves depicted in Figure 3. The figure depicts the case $k=3$; in general $\delta_{k}$ has $k$ twists around the upper right handle. (Specifically, the curve $\delta_{k}$ is equal to $T_{a_{3}}^{k}\left(\delta_{0}\right)$, where $a_{3}$ is as in Figure 5.) The regular neighborhood of $\gamma \cup \delta_{k}$ is a lantern $L_{k}$, and we fix an identification of $L_{k}$ with our reference lantern $L$ by specifying that $\gamma$ and $\delta_{k}$ should correspond to $x y$ and $y z$ respectively. Let $f_{k} \in \operatorname{Mag}_{g}$ be the element corresponding under this identification to the mapping class $T_{\alpha} T_{\beta}^{-1}$ on $L$; it is easy to check using the lantern relation that $f_{k}$ is in fact $\left[T_{\gamma}^{-1}, T_{\delta_{k}}^{-1}\right]$. We will show that the images $\Psi\left(f_{k}\right)$ are linearly independent (over $\mathbb{Z}$ ). 


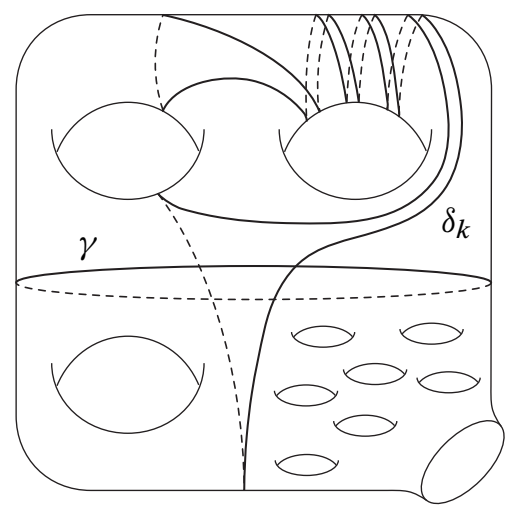

Figure 3: The curves $\gamma$ and $\delta_{k}$ for $k=3$

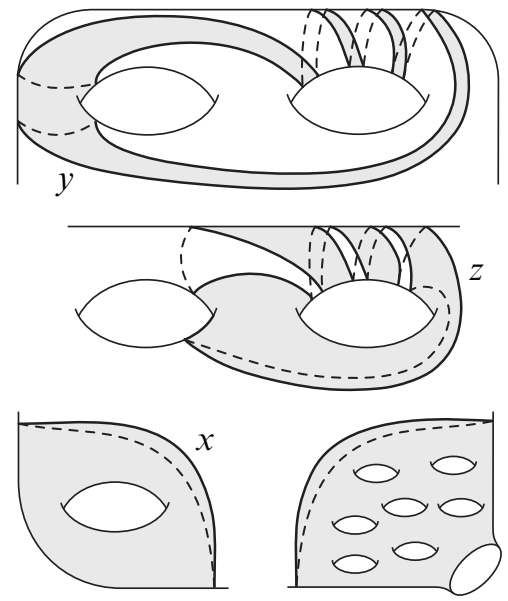

Figure 4: The boundary curves of $L_{k}$; the subsurfaces cut off by these curves are shaded

The boundary curves of $L_{k}$ are depicted in Figure 4.

With the basis $a_{1}, b_{1}, \ldots, a_{g}, b_{g}$ for $\pi_{1}\left(S_{g, 1}\right)$ as illustrated in Figure 5, we see that as curves $x, y$ and $z$ can be represented by $\left[a_{1}, b_{1}\right],\left[a_{2}, b_{3} a_{3}^{k} b_{2}\right]$ and $\left[b_{2} a_{2}^{-1} b_{2}^{-1} a_{3}, b_{3} a_{3}^{k}\right]$ respectively. As based loops, we actually have the conjugate $z={ }^{c}\left[b_{2} a_{2}^{-1} b_{2}^{-1} a_{3}, b_{3} a_{3}^{k}\right]$, where $c=\left[b_{3}, a_{3}\right]\left[b_{2}, a_{2}\right] a_{2}$. Note that with this representative for $z$, we have $x y z=\left[a_{1}, b_{1}\right]\left[a_{2}, b_{2}\right]\left[a_{3}, b_{3}\right]$, the fourth boundary curve in Figure 4 . 


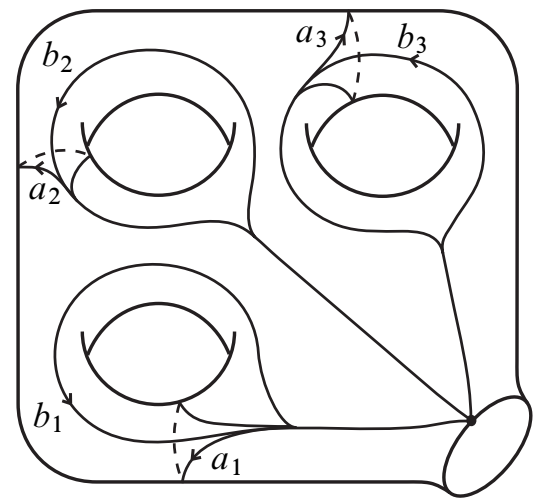

Figure 5: A basis for $\pi_{1}\left(S_{g, 1}\right)$

Note that $a_{1}$ and $a_{2}$ intersect each $L_{k}$ in arcs corresponding to $A_{1}$ and $A_{2}$. Thus by Proposition 3.1, we have

$\Psi\left(f_{k}\right)\left(\left\{a_{1}, a_{2}\right\}\right)=$

$$
\left(a_{1}-1\right)\left(a_{2}-1\right)\left[\left(\left\{a_{1}, b_{1}\right\}+\left\{a_{2}, b_{3} a_{3}^{k} b_{2}\right\}\right) \wedge a_{2}\left\{b_{2} a_{2}^{-1} b_{2}^{-1} a_{3}, b_{3} a_{3}^{k}\right\}\right] .
$$

Denote this element of $\bigwedge^{2} G^{\text {ab }}$ by $\alpha_{k}$. We now check that the elements $\left\{\alpha_{k}\right\}$ are linearly independent as follows. There is a standard embedding $G^{\text {ab }} \hookrightarrow(\mathbb{Z} H)^{2 g}$ given by sending the class $[x]$ to $\left(\partial x / \partial z_{1}, \ldots, \partial x / \partial z_{2 g}\right)$, where $\left\{z_{i}\right\}$ is our basis for $\pi_{1}(S)=F_{2 g}$ and where $\partial / \partial z_{i}$ are the Fox derivatives (see, for example, ChurchPixton [3] for a detailed explanation of this embedding). The only property of this embedding that we will need is that the elements below, which together make up $\alpha_{k}$, are mapped as follows by the embedding. Here the $A_{i}$ and $B_{i}$ make up a $\mathbb{Z} H-$ basis for $(\mathbb{Z} H)^{2 g}$.

$$
\begin{aligned}
&\left\{a_{1}, b_{1}\right\} \mapsto\left(1-b_{1}\right) A_{1}-\left(1-a_{1}\right) B_{1} \\
&\left\{a_{2}, b_{3} a_{3}^{k} b_{2}\right\} \mapsto\left(1-b_{3} a_{3}^{k} b_{2}\right) A_{2} \\
&-\left(1-a_{2}\right)\left(B_{3}+b_{3}\left(1+\cdots+a_{3}^{k-1}\right) A_{3}+b_{3} a_{3}^{k} B_{2}\right) \\
&\left\{b_{2} a_{2}^{-1} b_{2}^{-1} a_{3}, b_{3} a_{3}^{k}\right\} \mapsto\left(1-b_{3} a_{3}^{k}\right)\left(\left(1-a_{2}^{-1}\right) B_{2}-a_{2}^{-1} b_{2} A_{2}+a_{2}^{-1} A_{3}\right) \\
&-\left(1-a_{2}^{-1} a_{3}\right)\left(B_{3}+b_{3}\left(1+\cdots+a_{3}^{k-1}\right) A_{3}\right)
\end{aligned}
$$

By expanding out $\alpha_{k}$, we see that $\alpha_{N}$ is the only such element which contains the term $A_{1} \wedge b_{3} a_{3}^{N} B_{2}$ with nonzero coefficient; it follows that the $\alpha_{k}$ are linearly independent, as desired. 
As the image of $\Psi$ is abelian, Theorem 3.2 immediately implies Theorem 1.1 for $g \geq 3$. Note that the proof of Theorem 3.2 used in an essential way that $g \geq 3$. So in order to complete the proof of Theorem 1.1, we need another argument when $g=2$.

Theorem 3.3 $H_{1}\left(\mathrm{Mag}_{2}\right)$ has infinite rank; in fact, $\mathrm{Mag}_{2}$ surjects to a free group of infinite rank.

Proof Suzuki showed that the element $f=\left[T_{\gamma}, T_{\delta}\right]$ is in $\operatorname{Mag}_{2}$ for $\gamma$ and $\delta$ as in Figure 6; in particular $\mathrm{Mag}_{2}$ is nontrivial. Let $S_{2}$ be a closed surface of genus 2; we denote by $\mathcal{I}_{2, *}$ the Torelli group of $S_{2}$ with respect to a marked point $*$, and by $\mathcal{I}_{2}$ the Torelli group of the closed surface $S_{2}$. By Johnson [6], we have the exact sequence

$$
1 \longrightarrow \mathbb{Z} \longrightarrow \mathcal{I}_{2,1} \stackrel{p}{\longrightarrow} \mathcal{I}_{2, *} \longrightarrow 1,
$$

where the kernel is generated by a twist $T_{\omega}$ around the boundary $\omega=\partial S_{2}$. It is easy to check that the action of $T_{\omega}$ on $\pi_{1}\left(S_{2,1}\right)$ is conjugation by $\omega$; since $\omega \notin \Gamma^{3}$, we see that $T_{\omega} \notin \operatorname{Mag}_{2}$.

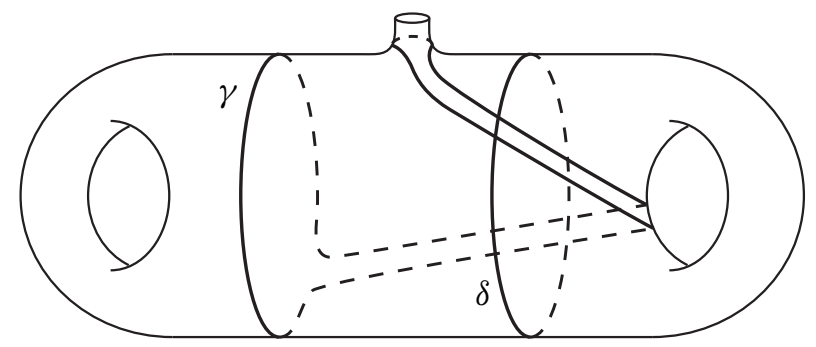

Figure 6: The commutator $\left[T_{\gamma}, T_{\delta}\right]$ lies in $\mathrm{Mag}_{2}$

It follows that $p$ restricts to an isomorphism between $\operatorname{Mag}_{2}$ and a subgroup $p\left(\operatorname{Mag}_{2}\right)<$ $\mathcal{I}_{2, *}$.

Again by Johnson [6], we have the exact sequence

$$
1 \longrightarrow \Lambda \longrightarrow \mathcal{I}_{2, *} \stackrel{\pi}{\longrightarrow} \mathcal{I}_{2} \longrightarrow 1,
$$

where $\Lambda \approx \pi_{1}\left(S_{2}, *\right)$; note that $\mathcal{I}_{2, *}$ acts on $\pi_{1}\left(S_{2}, *\right)$, and the restriction to $\Lambda$ is just the action by conjugation. Mess [8] proved that $\mathcal{I}_{2}$ is free of infinite rank. It is easy to see from Figure 6 that $f$ lies in $\operatorname{ker} \pi=\Lambda$. We use the following well-known lemma.

Lemma 3.4 Any nontrivial infinite index normal subgroup of a surface group or free group is an infinite rank free group. 
If the image $\pi \circ p\left(\operatorname{Mag}_{2}\right)<\mathcal{I}_{2} \approx F_{\infty}$ is nontrivial, it is an infinite rank free group; it either has finite index in $F_{\infty}$ and thus infinite rank, or infinite index, in which case Lemma 3.4 applies. Thus $\mathrm{Mag}_{2}$ surjects to the infinite rank free group $\pi \circ p\left(\operatorname{Mag}_{2}\right)$, and we are done.

Otherwise $p\left(\operatorname{Mag}_{2}\right) \subset \operatorname{ker} \pi=\Lambda$. Any $\varphi \in \operatorname{Mag}_{2}$ acts trivially on $\Gamma / \Gamma^{3} ;$ thus $p(\varphi)$ acts trivially on $\pi_{1}\left(S_{2}\right) / \pi_{1}\left(S_{2}\right)^{3}$. Since the action of $\Lambda$ is by conjugation, this implies that $p(\varphi)$ lies in $\Lambda^{3}$. Thus $p\left(\operatorname{Mag}_{2}\right)$ has infinite index in $\Lambda$, and so by Lemma 3.4, $p\left(\operatorname{Mag}_{2}\right) \approx \operatorname{Mag}_{2}$ is an infinite rank free group.

Theorem 1.1, and hence Corollary 1.2, follows immediately from Theorems 3.2 and 3.3 .

Remark One can check by explicit computation that for Suzuki's element $f \in \mathrm{Mag}_{2}$ above, $\Psi(f)=0$. It would be interesting to know whether $\Psi$ in fact vanishes on $\operatorname{Mag}_{2}$.

\section{Computing the image of $\Phi$}

The kernel $K$ of the map from $F_{n}=\left\langle x_{1}, \ldots, x_{n}\right\rangle$ to $\mathbb{Z}=\langle t\rangle$ which sends each $x_{i} \mapsto t$ is normally generated by the elements $x_{i} x_{j}^{-1}$. If we set $x_{i, k}:=x_{1}^{k} x_{i} x_{1}^{-k-1}$ for $i \neq 1$ and $k \in \mathbb{Z}$, then $\left\{x_{i, k}\right\}$ gives a basis for $K$ as a free group. As above, the action of $F_{n}$ on $K$ by conjugation descends to a $\mathbb{Z}\left[t, t^{-1}\right]$ action on $K^{\mathrm{ab}}$. With respect to this action we have $x_{i, k}=t^{k} x_{i, 0}$, and thus $K^{\mathrm{ab}}$ is a free $\mathbb{Z}\left[t, t^{-1}\right]$-module with basis $\left\{y_{i}=x_{i, 0}\right\}_{i \neq 1}$.

The braid group $B_{n}$ has generators $\sigma_{1}, \ldots, \sigma_{n-1}$; the action of $\sigma_{i}$ on $F_{n}$ sends $x_{i} \mapsto x_{i} x_{i+1} x_{i}^{-1}, x_{i+1} \mapsto x_{i}$, and fixes the other generators. The action of $B_{n}$ on $K^{\mathrm{ab}}$ commutes with the $\mathbb{Z}\left[t, t^{-1}\right]$ action.

Theorem 4.1 The image of $\Phi$ has infinite rank for $n \geq 6$.

Proof The element of Bur $_{6}$ found by Bigelow in [1] is the commutator of the halftwists along the arcs displayed in Figure 7. In terms of the Artin generators, this is

$$
\phi_{B}=\left[\psi_{1} \sigma_{3}^{-1} \psi_{1}^{-1}, \psi_{2} \sigma_{3}^{-1} \psi_{2}\right]
$$

where

$$
\psi_{1}=\sigma_{4} \sigma_{5}^{-1} \sigma_{2}^{-1} \sigma_{1} \quad \text { and } \quad \psi_{2}=\sigma_{4}^{-1} \sigma_{5}^{2} \sigma_{2} \sigma_{1}^{-1} .
$$

In Appendix A, we give the computation of $\alpha:=\Phi\left(\phi_{B}\right)\left(\left[x_{2} x_{1}^{-1}\right]\right)=\Phi\left(\phi_{B}\right)\left(y_{2}\right)$; it has 262 terms. The only fact about $\alpha$ that we will need is that its highest term of the form 


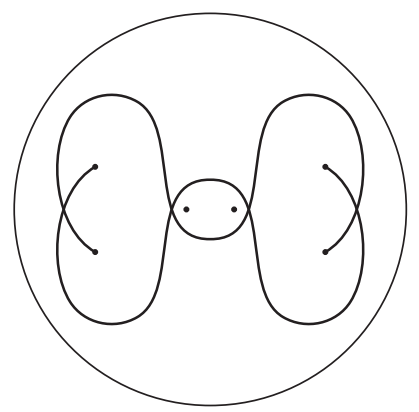

Figure 7: The two arcs defining Bigelow's element $\phi_{B}$

$y_{2} \wedge t^{j} y_{4}$ is $-2 y_{2} \wedge t^{3} y_{4}$, and its highest term of the form $y_{2} \wedge t^{j} y_{5}$ is $+2 y_{2} \wedge t^{2} y_{5}$ (these terms are set in boxes in the appendix).

It is easy to check that

$$
\begin{aligned}
\sigma_{4}^{2}\left(x_{4}\right) & =x_{4} x_{5} x_{4} x_{5}^{-1} x_{4}^{-1} \\
\sigma_{4}^{2}\left(x_{5}\right) & =x_{5} x_{4} x_{5}^{-1} \\
\sigma_{4}^{2}\left(x_{i}\right) & =x_{i} \text { for } i \neq 4,5 .
\end{aligned}
$$

By induction, for $k \geq 1$ we have

$$
\begin{aligned}
& \sigma_{4}^{2 k}\left(x_{4}\right)=\left(x_{4} x_{5}\right)^{k} x_{4}\left(x_{4} x_{5}\right)^{-k} \\
& \sigma_{4}^{2 k}\left(x_{5}\right)=\left(x_{4} x_{5}\right)^{k-1} x_{4} x_{5} x_{4}^{-1}\left(x_{4} x_{5}\right)^{k-1} \\
& \sigma_{4}^{2 k}\left(x_{i}\right)=x_{i} \text { for } i \neq 4,5 .
\end{aligned}
$$

The action of $\sigma_{4}^{2 k}$ on $K^{\mathrm{ab}}$ in terms of our basis is thus given by:

$$
\begin{aligned}
y_{4} & \mapsto\left(1-t+t^{2}-\cdots-t^{k-1}+t^{k}\right) y_{4}+\left(t-t^{2}+\cdots+t^{k-1}-t^{k}\right) y_{5} \\
y_{5} & \mapsto\left(1-t+t^{2}-\cdots-t^{k-1}\right) y_{4}+\left(t-t^{2}+\cdots+t^{k-1}\right) y_{5} \\
y_{i} & \mapsto y_{i} \quad \text { for } i \neq 4,5
\end{aligned}
$$

Now for $k \geq 0$ set

$$
\alpha_{k}:=\Phi\left(\sigma_{4}^{2 k} \phi_{B} \sigma_{4}^{-2 k}\right)\left(y_{2}\right) .
$$

By the equivariance of $\Phi$, and since $\sigma_{4}$ fixes $y_{2}$, we have $\alpha_{k}=\sigma_{4}^{2 k} \cdot \alpha$. From the action of $\sigma_{4}^{2 k}$ on $K^{\mathrm{ab}}$, we can see that the highest term in $\alpha_{N}$ of the form $y_{2} \wedge t^{j} y_{4}$ will be $-2 y_{2} \wedge t^{3+N} y_{4}$. Thus $\alpha_{N}$ is not contained in the span of $\left\{\alpha_{1}, \ldots, \alpha_{N-1}\right\}$; it follows that the $\alpha_{k}$ are linearly independent over $\mathbb{Z}$, and thus the image of $\Phi$ has infinite rank. 
Theorem 1.3 follows immediately.

\section{Appendix A Appendix}

The following computation was made, with the method explained in Section 4, with the help of Mathematica. A Mathematica notebook implementing these computations can be found at http://math.uchicago.edu/ tchurch/infinitegeneration.html or from the abstract page for this article.

The output of this notebook is $\Phi\left(\phi_{B}\right)\left(y_{2}\right)$, which is:

$$
\begin{aligned}
& -t^{-3} y_{2} \wedge t^{-2} y_{2} \quad+t^{-3} y_{2} \wedge t^{-1} y_{2} \\
& -t^{-3} y_{2} \wedge y_{2} \\
& -t^{-2} y_{2} \wedge y_{2} \\
& +t^{-1} y_{2} \wedge y_{2} \\
& +t^{-2} y_{2} \wedge t y_{2} \\
& -t^{3} y_{2} \wedge t^{4} y_{2} \\
& +t^{-1} y_{2} \wedge t y_{2} \\
& +t^{-2} y_{2} \wedge t^{-2} y_{3} \\
& +t^{-3} y_{2} \wedge t^{-4} y_{3} \\
& -2 y_{2} \wedge t^{2} y_{2} \\
& +t y_{2} \wedge t^{3} y_{2} \\
& +t^{2} y_{2} \wedge t^{3} y_{2} \\
& -t^{-1} y_{2} \wedge t^{-2} y_{3} \\
& -2 t^{-2} y_{2} \wedge y_{3} \\
& -2 y_{2} \wedge t^{2} y_{3} \\
& -t^{-2} y_{2} \wedge t^{-4} y_{3} \\
& -t^{-3} y_{2} \wedge t^{-3} y_{3} \\
& +t^{-1} y_{2} \wedge t^{-3} y_{3} \\
& -t^{2} y_{2} \wedge t^{-1} y_{3} \\
& -t^{-1} y_{3} \wedge t y_{3} \\
& +t^{4} y_{2} \wedge t^{3} y_{3} \\
& -y_{3} \wedge t^{3} y_{3} \\
& -t^{-2} y_{2} \wedge t^{-3} y_{4} \\
& +t^{-3} y_{2} \wedge y_{4} \\
& +t y_{3} \wedge y_{4} \\
& +t^{3} y_{3} \wedge t y_{4} \\
& -y_{4} \wedge t^{2} y_{4} \\
& +y_{4} \wedge t^{3} y_{4} \\
& -t^{2} y_{3} \wedge t^{4} y_{4} \\
& -t^{-2} y_{2} \wedge t^{-3} y_{5} \\
& +t^{-3} y_{3} \wedge t^{-2} y_{5} \\
& +t^{-3} y_{2} \wedge t^{-1} y_{3} \\
& -y_{2} \wedge t^{-1} y_{3} \\
& +t^{-1} y_{3} \wedge y_{3} \\
& +t y_{2} \wedge t^{-1} y_{3} \\
& +t^{3} y_{2} \wedge y_{3} \\
& -t^{4} y_{2} \wedge t^{2} y_{3} \\
& +t^{-1} y_{3} \wedge t^{2} y_{3} \\
& +2 t^{-1} y_{2} \wedge t y_{3} \\
& +t y_{3} \wedge t^{3} y_{3} \\
& -t^{2} y_{3} \wedge t^{3} y_{3} \\
& -t^{-3} y_{2} \wedge t^{-2} y_{4}+t^{-1} y_{2} \wedge t^{-2} y_{4}+t^{-2} y_{2} \wedge t^{-1} y_{4} \\
& -y_{2} \wedge y_{4} \\
& +t y_{2} \wedge y_{4} \\
& -t^{2} y_{3} \wedge y_{4} \\
& -2 t^{-2} y_{2} \wedge t y_{4} \\
& -t^{2} y_{2} \wedge y_{4} \\
& +y_{4} \wedge t y_{4}+2 t^{-1} y_{2} \wedge t^{2} y_{4} \\
& +t^{3} y_{2} \wedge t y_{4} \\
& -t^{-1} y_{3} \wedge t^{2} y_{4} \\
& \begin{array}{r}
-2 y_{2} \wedge t^{3} y_{4} \\
+t y_{2} \wedge t^{4} y_{4}
\end{array} \\
& -t^{4} y_{2} \wedge t^{3} y_{4} \\
& +t^{-1} y_{3} \wedge t^{3} y_{4} \\
& +t y_{2} \wedge t^{3} y_{3} \\
& +t^{-3} y_{2} \wedge t^{-3} y_{4} \\
& -t^{-3} y_{5} \wedge t^{-2} y_{5} \\
& -t y_{4} \wedge t^{4} y_{4} \\
& +t^{4} y_{2} \wedge t^{4} y_{4} \\
& +t^{2} y_{4} \wedge t^{4} y_{4} \\
& +t^{-3} y_{2} \wedge t^{-2} y_{5} \quad-t^{-2} y_{2} \wedge t^{-2} y_{5} \\
& -t^{-1} y_{3} \wedge t^{-2} y_{5} \\
& -t^{-3} y_{4} \wedge t^{-2} y_{5} \\
& -2 t^{-3} y_{2} \wedge t^{-1} y_{5} \\
& +t^{-1} y_{2} \wedge t^{-1} y_{5} \\
& +t^{-4} y_{3} \wedge t^{-1} y_{5} \\
& +2 t y_{4} \wedge t^{-1} y_{5} \\
& -y_{2} \wedge y_{5} \\
& -t y_{3} \wedge y_{5} \\
& -t^{-2} y_{3} \wedge t^{-1} y_{5} \\
& +2 y_{3} \wedge t^{-1} y_{5} \\
& +t^{-3} y_{2} \wedge y_{5} \\
& -t^{-3} y_{3} \wedge y_{5} \\
& -t^{2} y_{2} \wedge y_{5} \\
& -t^{2} y_{3} \wedge y_{5} \\
& -t^{-2} y_{4} \wedge y_{5} \\
& +t^{-1} y_{5} \wedge y_{5} \\
& -t^{3} y_{4} \wedge y_{5} \\
& +t^{3} y_{2} \wedge t y_{5} \\
& -y_{3} \wedge t^{4} y_{4} \\
& -t^{3} y_{4} \wedge t^{4} y_{4} \\
& +y_{2} \wedge t^{-2} y_{5} \\
& +t^{-2} y_{4} \wedge t^{-2} y_{5} \\
& +y_{2} \wedge t^{-1} y_{5} \\
& -t^{-1} y_{2} \wedge t^{-1} y_{4} \\
& -y_{3} \wedge y_{4} \\
& +t^{-1} y_{3} \wedge t y_{4} \\
& -t^{3} y_{3} \wedge t^{2} y_{4} \\
& +t^{3} y_{3} \wedge t^{3} y_{4} \\
& +t y_{3} \wedge t^{4} y_{4} \\
& +t^{-3} y_{2} \wedge t^{-3} y_{5} \\
& -t^{-4} y_{3} \wedge t^{-2} y_{5} \\
& -y_{4} \wedge t^{-2} y_{5} \\
& -t y_{2} \wedge t^{-1} y_{5} \\
& +t^{-3} y_{4} \wedge t^{-1} y_{5} \\
& +2 t^{-2} y_{2} \wedge y_{5} \\
& -t^{-1} y_{4} \wedge t^{-1} y_{5} \\
& -2 t^{-1} y_{2} \wedge y_{5} \\
& +t^{-2} y_{3} \wedge y_{5} \\
& +t^{-1} y_{4} \wedge y_{5} \\
& -t^{-3} y_{2} \wedge t y_{5} \\
& +t y_{2} \wedge t y_{5} \\
& +t^{3} y_{3} \wedge t y_{5} \\
& +y_{4} \wedge t y_{5} \\
& -y_{5} \wedge t y_{5} \\
& +t^{-1} y_{3} \wedge t y_{5} \\
& -y_{3} \wedge y_{5} \\
& -t y_{4} \wedge y_{5} \\
& -t^{-1} y_{2} \wedge t y_{5} \\
& -y_{3} \wedge t y_{5} \\
& -t y_{4} \wedge t y_{5} \\
& -t^{-2} y_{5} \wedge t y_{5} \\
& -t^{2} y_{2} \wedge t^{2} y_{5} \\
& +t^{3} y_{2} \wedge t^{2} y_{5} \\
& +t^{-2} y_{2} \wedge t^{2} y_{5} \\
& +2 t^{2} y_{4} \wedge t y_{5} \\
& \begin{array}{r}
+t^{4} y_{4} \wedge t y_{5} \\
+2 y_{2} \wedge t^{2} y_{5} \\
-y_{4} \wedge t^{2} y_{5}
\end{array} \\
& -t y_{2} \wedge t^{3} y_{5} \\
& -t^{3} y_{4} \wedge t^{2} y_{5} \\
& +t^{-1} y_{5} \wedge t^{2} y_{5} \\
& -t^{2} y_{2} \wedge t^{3} y_{5} \\
& -t^{4} y_{2} \wedge t^{3} y_{5} \\
& +t^{2} y_{2} \wedge t^{4} y_{5} \\
& +t^{2} y_{5} \wedge t^{3} y_{5} \\
& +t^{4} y_{4} \wedge t^{4} y_{5} \\
& -t^{-1} y_{3} \wedge t^{2} y_{5} \\
& -t^{-1} y_{2} \wedge t^{2} y_{5} \\
& -t^{2} y_{3} \wedge t^{2} y_{5} \\
& -2 y_{5} \wedge t^{2} y_{5} \\
& +y_{3} \wedge t^{3} y_{5} \\
& +t y_{5} \wedge t^{2} y_{5} \\
& +t y_{4} \wedge t^{3} y_{5} \\
& +t^{3} y_{2} \wedge t^{4} y_{5} \\
& -t^{2} y_{5} \wedge t^{4} y_{5} \\
& -t y_{3} \wedge t^{4} y_{5} \\
& +t^{3} y_{3} \wedge t^{4} y_{5} \\
& -t^{2} y_{4} \wedge t^{4} y_{5}
\end{aligned}
$$




$$
\begin{aligned}
& -t^{3} y_{2} \wedge t^{5} y_{5} \quad+t^{2} y_{3} \wedge t^{5} y_{5} \quad-t^{3} y_{3} \wedge t^{5} y_{5} \quad+t^{3} y_{4} \wedge t^{5} y_{5} \quad-t^{4} y_{4} \wedge t^{5} y_{5} \\
& +t^{3} y_{5} \wedge t^{5} y_{5}-t^{-3} y_{2} \wedge t^{-3} y_{6}+t^{-2} y_{2} \wedge t^{-3} y_{6}-t^{-2} y_{5} \wedge t^{-3} y_{6}+t^{-1} y_{5} \wedge t^{-3} y_{6} \\
& +t^{-3} y_{2} \wedge t^{-2} y_{6}-t^{-1} y_{2} \wedge t^{-2} y_{6}+t^{-2} y_{5} \wedge t^{-2} y_{6} \quad-y_{5} \wedge t^{-2} y_{6}+t^{-3} y_{2} \wedge t^{-1} y_{6} \\
& -t^{-2} y_{2} \wedge t^{-1} y_{6} \quad+y_{2} \wedge t^{-1} y_{6}-t^{-4} y_{3} \wedge t^{-1} y_{6}+t^{-3} y_{3} \wedge t^{-1} y_{6}-t^{-1} y_{3} \wedge t^{-1} y_{6} \\
& -t^{-3} y_{4} \wedge t^{-1} y_{6}+t^{-2} y_{4} \wedge t^{-1} y_{6} \quad-y_{4} \wedge t^{-1} y_{6}-t^{-3} y_{5} \wedge t^{-1} y_{6} \quad+t y_{5} \wedge t^{-1} y_{6} \\
& +t^{-3} y_{6} \wedge t^{-1} y_{6} \quad-t^{-2} y_{6} \wedge t^{-1} y_{6} \quad-t^{-3} y_{2} \wedge y_{6} \quad-t^{-2} y_{2} \wedge y_{6} \quad+t^{-1} y_{2} \wedge y_{6} \\
& +2 y_{2} \wedge y_{6} \quad-2 t y_{2} \wedge y_{6} \quad+t^{2} y_{2} \wedge y_{6} \quad+t^{-3} y_{3} \wedge y_{6} \quad-t^{-2} y_{3} \wedge y_{6} \\
& -t^{-1} y_{3} \wedge y_{6} \quad+3 y_{3} \wedge y_{6} \quad-t y_{3} \wedge y_{6} \quad+t^{2} y_{3} \wedge y_{6} \quad+t^{-2} y_{4} \wedge y_{6} \\
& -t^{-1} y_{4} \wedge y_{6} \quad-y_{4} \wedge y_{6} \quad+3 t y_{4} \wedge y_{6} \quad-t^{2} y_{4} \wedge y_{6} \quad+t^{3} y_{4} \wedge y_{6} \\
& -y_{5} \wedge y_{6} \quad+t y_{5} \wedge y_{6} \quad-2 t^{2} y_{5} \wedge y_{6} \quad-t^{-2} y_{6} \wedge y_{6} \quad+t^{-3} y_{2} \wedge t y_{6} \\
& +t^{-2} y_{2} \wedge t y_{6} \quad-y_{2} \wedge t y_{6} \quad-t y_{2} \wedge t y_{6} \quad-t^{3} y_{2} \wedge t y_{6} \quad-t^{-1} y_{3} \wedge t y_{6} \\
& +y_{3} \wedge t y_{6} \quad-2 t y_{3} \wedge t y_{6} \quad-t^{3} y_{3} \wedge t y_{6} \quad-y_{4} \wedge t y_{6} \quad+t y_{4} \wedge t y_{6} \\
& -2 t^{2} y_{4} \wedge t y_{6} \quad-t^{4} y_{4} \wedge t y_{6} \quad+t^{-2} y_{5} \wedge t y_{6} \quad+t^{-1} y_{5} \wedge t y_{6} \quad+t^{2} y_{5} \wedge t y_{6} \\
& +t^{3} y_{5} \wedge t y_{6} \quad+t^{-1} y_{6} \wedge t y_{6} \quad+2 y_{6} \wedge t y_{6} \quad-t^{-2} y_{2} \wedge t^{2} y_{6} \quad-t^{-1} y_{2} \wedge t^{2} y_{6} \\
& +t^{2} y_{2} \wedge t^{2} y_{6} \quad+t^{-1} y_{3} \wedge t^{2} y_{6} \quad+t^{2} y_{3} \wedge t^{2} y_{6} \quad+t^{3} y_{3} \wedge t^{2} y_{6} \quad+y_{4} \wedge t^{2} y_{6} \\
& +t^{3} y_{4} \wedge t^{2} y_{6} \quad+t^{4} y_{4} \wedge t^{2} y_{6} \quad-t^{-1} y_{5} \wedge t^{2} y_{6} \quad+t y_{5} \wedge t^{2} y_{6} \quad-t^{2} y_{5} \wedge t^{2} y_{6} \\
& -t^{4} y_{5} \wedge t^{2} y_{6} \quad-2 y_{6} \wedge t^{2} y_{6} \quad-t y_{6} \wedge t^{2} y_{6} \quad+2 y_{2} \wedge t^{3} y_{6} \quad+t^{4} y_{2} \wedge t^{3} y_{6} \\
& -t^{-1} y_{3} \wedge t^{3} y_{6} \quad-t^{3} y_{3} \wedge t^{3} y_{6} \quad-y_{4} \wedge t^{3} y_{6} \quad-t^{4} y_{4} \wedge t^{3} y_{6} \quad-y_{5} \wedge t^{3} y_{6} \\
& -t^{2} y_{5} \wedge t^{3} y_{6} \quad+t^{5} y_{5} \wedge t^{3} y_{6} \quad+y_{6} \wedge t^{3} y_{6} \quad+t^{2} y_{6} \wedge t^{3} y_{6} \quad-t y_{2} \wedge t^{4} y_{6} \\
& -t^{2} y_{2} \wedge t^{4} y_{6} \quad-t^{4} y_{2} \wedge t^{4} y_{6} \quad+y_{3} \wedge t^{4} y_{6} \quad+t y_{4} \wedge t^{4} y_{6} \quad+t y_{5} \wedge t^{4} y_{6} \\
& +t^{2} y_{5} \wedge t^{4} y_{6} \quad+t^{4} y_{5} \wedge t^{4} y_{6} \quad-t^{5} y_{5} \wedge t^{4} y_{6} \quad-t y_{6} \wedge t^{4} y_{6} \quad+t^{3} y_{2} \wedge t^{5} y_{6} \\
& -t^{2} y_{3} \wedge t^{5} y_{6} \quad+t^{3} y_{3} \wedge t^{5} y_{6} \quad-t^{3} y_{4} \wedge t^{5} y_{6} \quad+t^{4} y_{4} \wedge t^{5} y_{6} \quad-t^{3} y_{5} \wedge t^{5} y_{6} \\
& +t^{3} y_{6} \wedge t^{5} y_{6} \quad-t^{4} y_{6} \wedge t^{5} y_{6}
\end{aligned}
$$

\section{References}

[1] S Bigelow, The Burau representation is not faithful for $n=5$, Geom. Topol. 3 (1999) 397-404 MR1725480

[2] J S Birman, Braids, links, and mapping class groups, Annals of Mathematics Studies 82, Princeton University Press, Princeton, N.J. (1974) MR0375281

[3] T Church, A Pixton, Separating twists and the Magnus representation of the Torelli group arXiv:0804.3633

[4] R H Fox, Free differential calculus I: Derivation in the free group ring, Ann. of Math. (2) 57 (1953) 547-560 MR0053938

[5] D Johnson, An abelian quotient of the mapping class group $\mathcal{I}_{g}$, Math. Ann. 249 (1980) 225-242 MR579103

[6] D Johnson, The structure of the Torelli group I: A finite set of generators for $\mathcal{I}$, Ann. of Math. (2) 118 (1983) 423-442 MR727699

[7] D D Long, M Paton, The Burau representation is not faithful for $n \geq 6$, Topology 32 (1993) 439-447 MR1217079 
[8] G Mess, The Torelli groups for genus 2 and 3 surfaces, Topology 31 (1992) 775-790 MR1191379

[9] J A Moody, The Burau representation of the braid group $B_{n}$ is unfaithful for large $n$, Bull. Amer. Math. Soc. (N.S.) 25 (1991) 379-384 MR1098347

[10] S Morita, Structure of the mapping class groups of surfaces: a survey and a prospect, from: "Proceedings of the Kirbyfest (Berkeley, CA, 1998)", Geom. Topol. Monogr. 2, Geom. Topol. Publ., Coventry (1999) 349-406 MR1734418

[11] T Satoh, The cokernel of the Johnson homomorphisms of the automorphism group of a free metabelian group, Trans. Amer. Math. Soc. 361 (2009) 2085-2107 MR2465830

[12] M Suzuki, The Magnus representation of the Torelli group $\mathcal{I}_{g, 1}$ is not faithful for $g \geq 2$, Proc. Amer. Math. Soc. 130 (2002) 909-914 MR1866048

[13] M Suzuki, On the kernel of the Magnus representation of the Torelli group, Proc. Amer. Math. Soc. 133 (2005) 1865-1872 MR2120289

Department of Mathematics, 5734 S. University Ave., Chicago, IL 60637

tchurch@math.uchicago.edu, farb@math.uchicago.edu

Received: 28 October 2009 